\title{
WEIGHTED INTEGRAL REPRESENTATIONS IN TUBE DOMAIN OVER REAL UNIT BALL
}

\author{
ARMAN H. KARAPETYAN
}

\begin{abstract}
Weighted spaces of functions holomorphic in tube domain over the real unit ball of $R^{n}$ are considered. For these classes reproducing kernels are constructed in an explicit form. Estimates for these kernels are obtained.
\end{abstract}

\section{INTRODUCTION}

It is well known that the Hardy class $H^{2}(\operatorname{Re} z>0)$ is defined as the set of all holomorphic functions $f(z), \operatorname{Re} z>0$, satisfying the condition

$$
\sup _{x>0}\left(\int_{-\infty}^{+\infty}|f(x+i y)|^{2} d y\right)<+\infty .
$$

The following result was established by R.Paley and N.Wiener[1]:

Theorem 1.1. The class $H^{2}($ Rez $>0)$ admits an integral representation of the form

$$
f(z)=\int_{0}^{+\infty} F(t) e^{-z t} d t, \quad \operatorname{Re} z>0,
$$

where $F(t) \in L^{2}(0 ;+\infty)$.

Theorem 1.1 initiated numerous investigations where this classical result was generalized in various directions.

In [2] (see also [3, Chapter 7]) Hardy type weighted classes in arbitrary angular domains were introduced and for these classes Paley-Wiener type integral representations by means of Mittag-Leffler type kernels were established.

In [4] (see also [5]) an analogue of Theorem 1.1 for multidimensional Hardy classes $H^{2}$ over radial tube domains in $C^{n}$ were established. Later

2010 Mathematics Subject Classification. 32A07, 32A25, 32A36, 26D15, 42B10, 44A10.

Key words and phrases. Tube domains, weighted spaces of holomorphic functions, Paley-Wiener type theorems, reproducing kernels, weighted integral representations.

Copyright (c) 2016 by ANUBIH. 
on, in [6], this result was generalized for Siegel domains of type two (much more general than radial tube domains). Moreover, in $[6, \S 5]$ a somewhat different problem was set and solved: to obtain Paley-Wiener type integral representations for classes of functions holomorphic in Siegel domains of type two and belonging to $L^{2}$ over the whole domain. On the basis of these integral representations reproducing kernels for the classes were constructed in an explicit form.

In order to review further investigations we need some notations.

For any $z=\left(z_{1}, \ldots, z_{n}\right) \in C^{n}$ and $w=\left(w_{1}, \ldots, w_{n}\right) \in C^{n}$ set

$$
<z, w>=\sum_{k=0}^{n} z_{k} \overline{w_{k}} .
$$

Suppose that $B \subset R^{n}$ is a domain and $\gamma(y)>0, y \in B$, is an arbitrary continuous function. We put

$$
\gamma^{*}(t)=\int_{B} e^{-<y, t>} \gamma(y) d y, t \in R^{n}
$$

Further, for $p, s \in(0 ;+\infty)$ we denote by $H_{s, \gamma}^{p}\left(T_{B}\right)$ the set of all functions $f(z) \equiv f(x+i y)$ holomorphic in the tube domain

$$
T_{B}=\left\{z=x+i y \in C^{n}: x \in R^{n}, y \in B\right\}
$$

and satisfying the condition

$$
M_{s, \gamma}^{p}(f) \equiv \int_{B}\left(\int_{R^{n}}|f(x+i y)|^{p} d x\right)^{s} \gamma(y) d y<+\infty .
$$

For any $f \in H_{s, \gamma}^{p}\left(T_{B}\right)$ put

$$
I(f)=\left\{y \in B: f_{y}(x) \equiv f(x+i y) \in L^{p}\left(R^{n}\right)\right\} .
$$

Obviously, mes $\{B \backslash I(f)\}=0$.

Note that for $s=1$ the space $H_{s, \gamma}^{p}\left(T_{B}\right)=H_{1, \gamma}^{p}\left(T_{B}\right)$ consists of those functions holomorphic in $T_{B}$, which are from $L^{p}\left\{T_{B} ; \gamma(y) d x d y\right\}$.

The following theorem is valid:

Theorem 1.2. 1. Assume that $2 \leq p<+\infty, 1 / p+1 / q=1,0<s<+\infty$ and a measurable function $F(t), t \in R^{n}$, satisfies the condition

$$
\int_{B}\left(\int_{R^{n}}|F(t)|^{q} e^{-q<y, t>} d t\right)^{s(p-1)} \gamma(y) d y<+\infty .
$$

Then the function

$$
f(z)=\frac{1}{(2 \pi)^{n / 2}} \int_{R^{n}} F(t) e^{i<z, t>} d t, \quad z \in T_{B},
$$


belongs to the space $H_{s, \gamma}^{p}\left(T_{B}\right)$ and

$$
M_{s, \gamma}^{p}(f) \leq \frac{1}{(2 \pi)^{\frac{n}{2} s(p-2)}} \int_{B}\left(\int_{R^{n}}|F(t)|^{q} e^{-q<y, t>} d t\right)^{s(p-1)} \gamma(y) d y<+\infty .
$$

2. Assume that $1 \leq p \leq 2$ and $0<s<+\infty$. Then each function $f \in$ $H_{s, \gamma}^{p}\left(T_{B}\right)$ admits an integral representation of the form (1.9), where:

- if $p=1, F(t), t \in R^{n}$ is continuous and satisfies the condition

$$
\sup _{t \in R^{n}}\left\{|F(t)| \gamma^{*}(s t)\right\} \leq \frac{M_{s, \gamma}^{1}(f)}{(2 \pi)^{\frac{n}{2} s}}<+\infty ;
$$

- if $1<p \leq 2, F(t), t \in R^{n}$, is measurable and satisfies the condition

$$
\begin{aligned}
\int_{B}\left(\int_{R^{n}}|F(t)|^{q} e^{-q<y, t>} d t\right)^{s(p-1)} \gamma(y) d y & \\
\leq & \frac{M_{s, \gamma}^{p}(f)}{(2 \pi)^{\frac{n}{2} s(2-p)}}<+\infty \quad(1 / p+1 / q=1) .
\end{aligned}
$$

Also, for any $y \in I(f)$ we have

$$
\hat{f}_{y}(t)=F(t) e^{-<y, t>}, \quad t \in R^{n},
$$

where $\hat{f}_{y}$ is the Fourier transform of $f_{y}(x), x \in R^{n}$.

3. For $p=2$ and $0<s<+\infty$ the formula (1.9) gives an integral representation of the whole class $H_{s, \gamma}^{p}\left(T_{B}\right)$, i.e. $H_{s, \gamma}^{2}\left(T_{B}\right)$ coincides with the set of all functions $f(z)$ representable in the form (1.9) with a measurable function $F(t), t \in R^{n}$, satisfying the condition

$$
\int_{B}\left(\int_{R^{n}}|F(t)|^{2} e^{-2<y, t>} d t\right)^{s} \gamma(y) d y<+\infty .
$$

Moreover, the Parseval identity holds:

$$
M_{s, \gamma}^{2}(f)=\int_{B}\left(\int_{R^{n}}|F(t)|^{2} e^{-2<y, t>} d t\right)^{s} \gamma(y) d y .
$$

For $n \geq 1, \gamma(y) \equiv 1(y \in B)$ and $p=2, s=1$ Theorem 1.2 follows from results of [6] formulated for arbitrary Siegel domains.

For $n \geq 1, \gamma(y) \equiv 1(y \in B)$ and under assumptions $1 \leq p \leq 2, s=1$ or $1<p<2, s=1 /(p-1)$ the formulated assertions were established in [7-9].

For $n=1, \gamma(y) \equiv 1(y \in B)$ the theorem follows from more general results of $[10]$.

Finally, in the form formulated above, Theorem 1.2 was established in [11, Theorems 2.3-2.5] and [12, Theorem 2].

It turns out [13-15] that the supports of functions $F(t)$ in Theorem 1.2 $(2,3)$ are significantly narrow if only the domain $B \subset R^{n}$ is a sharp (or 
an acute) open convex cone $V \subset R^{n}$ and the weight function $\gamma(y),(y \in$ $V)$ satisfy certain growth conditions adapted to the structure of $V$. In all these cases we have $\operatorname{supp} F(t) \subset V^{*}$, where $V^{*}$ is a cone conjugate to $V$. Furthermore, in[13-15] for $1 \leq p \leq 2$ and under additional conditions on $\gamma(y), y \in V$ and on the parameter $s$ a reproducing kernel for the class $H_{s, \gamma}^{p}\left(T_{V}\right)$ was constructed. In [16] similar problems were solved in tube domains over polyhedra $P \subset R^{n}$.

In [17-19], in a sense, a converse problem was considered and solved: given a domain $D \subset R^{n}$, integrals of the form

$$
f(z)=\frac{1}{(2 \pi)^{n / 2}} \int_{D} F(t) e^{i<z, t>} d t,
$$

were considered for, in general, arbitrary functions $F(t), t \in D$. Then, requiring the holomorphic functions $f(z)$ to belong to Hardy type spaces (including the condition of square integrability over domain), the corresponding conditions on functions $F(t)$ and the maximal tube domain in $C^{n}$ where the functions (1.16) can be extended, were determined. For the considered spaces reproducing kernels were constructed.

It should be mentioned that for the case $p=2, s=1, \gamma(y) \equiv 1$ the results of [11-15] are close to [17-19].

In the present paper we consider above-mentioned spaces $H_{s, \gamma}^{p}\left(T_{B}\right)$ where the domain $B$ is the unit ball in the space $R^{n}$. For these spaces the corresponding reproducing kernels are constructed (in an explicit form) and estimated.

\section{Main integral RePRESEntations}

Assume that $1 \leq p \leq 2,2 \leq q \leq+\infty$ and $1 / p+1 / q=1$. It is well-known that for a function $f \in L^{p}\left(R^{n}\right)$ its Fourier transform is defined as follows:

$$
\hat{f}(t)=\frac{1}{(2 \pi)^{n / 2}} \int_{R^{n}} f(x) e^{-i<t, x>} d x, t \in R^{n},
$$

if $p=1$, and

$$
\hat{f}(t)=\text { l.i.m. } A \rightarrow+\infty \frac{1}{(2 \pi)^{n / 2}} \int_{|x|<A} f(x) e^{-i<t, x>} d x, t \in R^{n},
$$

if $1<p \leq 2$, where the l.i.m. is understood in the sense of the space $L^{q}\left(R^{n}\right)$.

In what follows we need the following two important (and well-known) assertions:

Theorem 2.1. For any functions $f_{1}, f_{2} \in L^{p}\left(R^{n}\right), 1 \leq p \leq 2$ we have

$$
\int_{R^{n}} \hat{f}_{1}(x) \cdot f_{2}(x) d x=\int_{R^{n}} f_{1}(x) \cdot \hat{f}_{2}(x) d x .
$$


Theorem 2.2. (Hausdorff-Young theorem) For any function $f \in L^{p}\left(R^{n}\right)$, $1 \leq p \leq 2$ we have

$$
\left\{\frac{1}{(2 \pi)^{n / 2}} \cdot \int_{R^{n}}|\hat{f}(t)|^{q} d t\right\}^{1 / q} \leq\left\{\frac{1}{(2 \pi)^{n / 2}} \cdot \int_{R^{n}}|f(x)|^{p} d x\right\}^{1 / p} .
$$

In what follows we suppose that $B$ is the unit ball in the space $R^{n}$, i.e. $B=\left\{y \in R^{n}:|y|<1\right\}$. Moreover, $\gamma(y), y \in B$, is supposed to be a positive continuous function such that $\gamma \in L^{1}(B ; d y)$. Naturally, $\gamma^{*}(t), t \in R^{n}$, is defined by the formula (1.4). Denote by $S$ the unit sphere in $R^{n}$ and let $\sigma$ be the surface Lebesgue measure on $S$.

Proposition 2.1. The function $\gamma^{*}(t), t \in R^{n}$, has the following properties:

(a) $0<\gamma^{*}(t)<+\infty$ for any $t \in R^{n}$;

(b) $\gamma^{*}(t), t \in R^{n}$, is a continuous function;

(c) There exists a constant $C_{1}=C_{1}(\gamma)$ such that

$$
\gamma^{*}(t) \leq C_{1} \cdot e^{|t|}, \forall t \in R^{n}
$$

(d) For any $\delta \in(0 ; 1)$ there exists a constant $C_{2}(\delta ; \gamma ; n)$ such that

$$
\gamma^{*}(t) \geq C_{2} \cdot e^{(1-\delta)|t|}, \forall t \in R^{n} .
$$

Proof. Note that

$$
e^{-<y, t>} \cdot \gamma(y) \leq e^{|t|} \cdot \gamma(y)
$$

uniformly in $y \in B$ and $t \in R^{n}$. Hence

$$
\gamma^{*}(t) \leq e^{|t|} \cdot \int_{B} \gamma(y) d y \equiv C_{1}(\gamma) \cdot e^{|t|}<+\infty, \forall t \in R^{n} .
$$

Thus, (c) and (a) are proved. By the way, in view of (2.7), (b) is also proved. Further, assume that $t \neq 0 \Rightarrow t=|t| \cdot t_{0}$ with $t_{0} \in S$ (i.e. $\left.\left|t_{0}\right|=1\right)$. The passage to polar coordinates $\left(y=r \cdot \zeta, r>0, \zeta \in S \Rightarrow d y=r^{n-1} d r d \sigma(\zeta)\right)$ yields:

$$
\begin{aligned}
\gamma^{*}(t) & =\int_{0}^{1} r^{n-1} \int_{S} e^{-r<\zeta, t>} \cdot \gamma(r \cdot \zeta) d \sigma(\zeta) d r \\
& =\int_{0}^{1} r^{n-1} \int_{S} e^{-r|t|<\zeta, t_{0}>} \cdot \gamma(r \cdot \zeta) d \sigma(\zeta) d r .
\end{aligned}
$$

For any $\eta \in(0 ; 1)$ there exists a neighborhood $G \subset S$ of $-t_{0} \in S$ such that

$$
<\zeta,-t_{0}>\geq 1-\eta \Leftrightarrow-<\zeta, t_{0}>\geq 1-\eta, \forall \zeta \in G .
$$


Note that mes $(G)$ depends on $\eta$ and $n$, but does not depend on $t_{0} \in S$.

$$
\begin{aligned}
\gamma^{*}(t) & \geq \int_{0}^{1} r^{n-1} \int_{G} e^{-r|t|<\zeta, t_{0}>} \cdot \gamma(r \cdot \zeta) d \sigma(\zeta) d r \\
& \geq \int_{0}^{1} r^{n-1} e^{r|t|(1-\eta)} \cdot \int_{G} \gamma(r \cdot \zeta) d \sigma(\zeta) d r .
\end{aligned}
$$

Now fix also an arbitrary $\varepsilon \in(0 ; 1)$, then

$$
\begin{aligned}
\gamma^{*}(t) & \geq \int_{1-\varepsilon}^{1} r^{n-1} e^{r|t|(1-\eta)} \cdot \int_{G} \gamma(r \cdot \zeta) d \sigma(\zeta) d r \\
& \geq e^{(1-\varepsilon)(1-\eta)|t|} \cdot \int_{1-\varepsilon}^{1} r^{n-1} \int_{G} \gamma(r \cdot \zeta) d \sigma(\zeta) d r \\
& =e^{(1-\varepsilon)(1-\eta)|t|} \cdot \int_{1-\varepsilon \leq r<1, \zeta \in G} \gamma(y) d y
\end{aligned}
$$

Note that

$$
\int_{1-\varepsilon \leq r<1, \zeta \in G} \gamma(y) d y \geq \operatorname{const}(\eta ; \varepsilon ; \gamma ; n)>0
$$

uniformly in $t_{0} \in S$. Hence

$$
\gamma^{*}(t) \geq \operatorname{const}(\eta ; \varepsilon ; \gamma ; n) \cdot e^{(1-\varepsilon)(1-\eta)|t|}, \forall t \in R^{n} .
$$

It remains to note that $\eta$ and $\varepsilon$ can be chosen in a such way that $(1-\varepsilon)(1-$ $\eta) \geq(1-\delta)$.

Proposition 2.2. For any $v, y \in B$ put

$$
\psi_{v, y}(t)=\frac{e^{-<v+y, t>}}{\gamma^{*}(2 t)}, t \in R^{n} .
$$

Then for any $\rho \in(0 ; 1)$ there exists a function $\psi$ belonging to all spaces $L^{p}\left(R^{n}\right), 0<p \leq \infty$ such that

$$
\psi_{v, y}(t) \leq \psi(t), \quad t \in R^{n}
$$

uniformly in $v$ with $|v|<1$ and $y$ with $|y| \leq \rho<1$.

Proof. Application of Proposition 2.1 yields $\left(t \in R^{n}\right)$ :

$$
\left|\psi_{v, y}(t)\right| \leq \frac{e^{(|v|+|y|)|t|}}{\gamma^{*}(2 t)} \leq \mathrm{const} \cdot \frac{e^{(1+\rho)|t|}}{e^{2(1-\delta)|t|}} \equiv \psi(t) \in L^{p}\left(R^{n}\right), \quad 0<p \leq \infty,
$$

if only $\delta>0$ is sufficiently small to ensure the condition $1+\rho<2(1-\delta)$.

For any $z=x+i y \in T_{B}, w=u+i v \in T_{B}$ put

$$
\Phi(z, w)=\int_{R^{n}} \frac{e^{i<z-\bar{w}, t>}}{\gamma^{*}(2 t)} d t
$$


where $\bar{w}$ is understood as $\left(\overline{w_{1}}, \overline{w_{2}}, \ldots, \overline{w_{n}}\right)$.

The properties of the kernel introduced are described by the following:

\section{Proposition 2.3.}

(a) The integral above converges absolutely, i.e. the kernel $\Phi$ is well defined for $\forall z=x+i y, w=u+i v \in T_{B}$;

(b) $\Phi(z, w)$ is holomorphic in $z \in T_{B}$ and antiholomorphic in $w \in T_{B}$;

(c) For fixed $z \in T_{B}$ and $v \in B, \Phi\left(z, u+i v\right.$ ) (as a function of $u \in R^{n}$ ) is the Fourier transform of the function

$$
(2 \pi)^{n / 2} \cdot \frac{e^{i<z+i v, t>}}{\gamma^{*}(2 t)}, t \in R^{n},
$$

which belongs to all spaces $L^{p}\left(R^{n}\right), 0<p \leq \infty$.

(d) If $0<\delta<2$, then

$$
|\Phi(z, w)| \leq \operatorname{const}(\delta ; \gamma ; n)
$$

uniformly in $z=x+i y, w=u+i v \in T_{B}$ with $|v|+|y| \leq 2-\delta$.

Proof. First of all, under the assumption $|v|+|y| \leq 2-\delta$ with any $\delta \in(0 ; 2)$ we have:

$$
\left|\frac{e^{i<z-\bar{w}, t>}}{\gamma^{*}(2 t)}\right| \leq \frac{e^{-<v+y, t>}}{\gamma^{*}(2 t)} \leq \frac{e^{(|v|+|y|)|t|}}{\gamma^{*}(2 t)} \leq \frac{e^{(2-|\delta|)|t|}}{\gamma^{*}(2 t)} .
$$

Further, according to Proposition 2.1,

$$
\begin{aligned}
\gamma^{*}(t) & \geq \operatorname{const}(\delta ; \gamma) \cdot e^{\left(1-\frac{\delta}{4}\right)|t|} \Rightarrow \\
\frac{1}{\gamma^{*}(2 t)} & \leq \operatorname{const}(\delta ; \gamma ; n) \cdot \frac{1}{e^{\left(2-\frac{\delta}{2}\right)|t|}}, \quad \forall t \in R^{n} .
\end{aligned}
$$

Hence

$$
\left|\frac{e^{i<z-\bar{w}, t>}}{\gamma^{*}(2 t)}\right| \leq \operatorname{const}(\delta ; \gamma ; n) \cdot \frac{e^{(2-|\delta|)|t|}}{e^{\left(2-\frac{\delta}{2}\right)|t|}}=\operatorname{const}(\delta ; \gamma ; n) \cdot e^{-\frac{\delta}{2}|t|}, t \in R^{n} .
$$

The last estimate immediately implies the assertions (a) and (b). To prove (c), it suffices to rewrite (2.17) as

$$
\Phi(z, w)=\frac{1}{(2 \pi)^{n / 2}} \int_{R^{n}}\left\{(2 \pi)^{n / 2} \cdot \frac{e^{i<z+i v, t>}}{\gamma^{*}(2 t)}\right\} \cdot e^{-i<u, t>} d t,
$$

and then note that the inner function

$$
(2 \pi)^{n / 2} \cdot \frac{e^{i<z+i v, t>}}{\gamma^{*}(2 t)} \in L^{p}\left(R^{n}\right), \quad 0<p \leq \infty,
$$

due to inequality (2.20). 
Finally, combination of (2.17) and (2.20) yields:

$$
\begin{gathered}
|\Phi(z, w)| \leq \operatorname{const}(\delta ; \gamma ; n) \cdot \int_{R^{n}} e^{-\frac{\delta}{2}|t|} d t \stackrel{\frac{\delta}{2} t \rightarrow t}{=}= \\
=\operatorname{const}(\delta ; \gamma ; n) \cdot \frac{1}{\delta^{n}} \cdot \int_{R^{n}} e^{-|t|} d t=\operatorname{const}(\delta ; \gamma ; n) \cdot \frac{1}{\delta^{n}} \equiv \operatorname{const}(\delta ; \gamma ; n) .
\end{gathered}
$$

The proof is completed.

Theorem 2.3. Assume that $1 \leq p \leq 2,1 / p \leq s<+\infty$, then each function $f \in H_{s, \gamma}^{p}\left(T_{B}\right)$ has the following integral representation $\left(z \in T_{B}\right)$ :

$$
f(z)=\frac{1}{(2 \pi)^{n}} \int_{T_{B}} f(w) \Phi(z, w) \gamma(v) d u d v, \quad(w=u+i v) .
$$

Proof. Let $f$ be a function from $H_{s, \gamma}^{p}\left(T_{B}\right)$. According to Theorem 1.2(2)

$$
f(z)=\frac{1}{(2 \pi)^{n / 2}} \int_{R^{n}} F(t) e^{i<z, t>} d t, \quad z \in T_{B},
$$

where $F(t), t \in R^{n}$, is continuous and satisfies (1.11) for $p=1$, and $F(t), t \in$ $R^{n}$, is measurable and satisfies (1.12) for $1<p \leq 2$. Moreover, for a.e. $v \in B$

$$
\hat{f}_{v}(t)=F(t) e^{-<v, t>}, t \in R^{n},
$$

where $f_{v}(u) \equiv f(u+i v), u \in R^{n}$.

Now fix an arbitrary $z=x+i y \in T_{B}$ and set

$$
I(z)=\frac{1}{(2 \pi)^{n}} \int_{T_{B}} f(w) \Phi(z, w) \gamma(v) d u d v .
$$

Assuming the absolute convergence of the integral (2.26), we consecutively use Proposition 2.3(c), Theorem 2.1, (2.25), (1.4), (2.24) and establish the the equality $I(z)=f(z)$ in the following way:

$$
\begin{aligned}
I(z) & =\frac{1}{(2 \pi)^{n}} \int_{B} \gamma(v) \int_{R^{n}} f(u+i v) \Phi(z, u+i v) d u d v \\
& =\frac{1}{(2 \pi)^{n}} \int_{B} \gamma(v) \int_{R^{n}} f_{v}(u)\left\{(2 \pi)^{n / 2} \cdot \frac{e^{i<z+i v, t>}}{\gamma^{*}(2 t)}\right\}(u) d u d v \\
& =\frac{1}{(2 \pi)^{n}} \int_{B} \gamma(v) \int_{R^{n}} \hat{f}_{v}(t)(2 \pi)^{n / 2} \cdot \frac{e^{i<z+i v, t>}}{\gamma^{*}(2 t)} d t d v \\
& =\frac{1}{(2 \pi)^{n / 2}} \int_{B} \gamma(v) \int_{R^{n}} F(t) e^{-<v, t>} \frac{e^{i<z+i v, t>}}{\gamma^{*}(2 t)} d t d v \\
& =\frac{1}{(2 \pi)^{n / 2}} \int_{R^{n}} F(t) \frac{e^{i<z, t>}}{\gamma^{*}(2 t)} \int_{B} \gamma(v) e^{-2<v, t>} d v d t
\end{aligned}
$$




$$
=\frac{1}{(2 \pi)^{n / 2}} \int_{R^{n}} F(t) e^{i<z, t>} d t=f(z) .
$$

So it remains to show that the integral $I(z)$ converges absolutely. To this end set

$$
\widetilde{I}(z)=\int_{B} \gamma(v) \int_{R^{n}}\left|f_{v}(u)\right||\Phi(z, u+i v)| d u d v .
$$

An application of Hölder's integral inequality gives:

$$
\int_{R^{n}}\left|f_{v}(u)\right||\Phi(z, u+i v)| d u \leq \widetilde{f}(v) \cdot\left(\int_{R^{n}}|\Phi(z, u+i v)|^{q} d u\right)^{1 / q}(1 / p+1 / q=1),
$$

where

$$
\widetilde{f}(v)=\left(\int_{R^{n}}\left|f_{v}(u)\right|^{p} d u\right)^{1 / p}, v \in B
$$

Further, the condition $1 \leq p \leq 2$ makes it possible an application of Hausdorff-Young theorem (Theorem 2.1):

$$
\begin{aligned}
\left(\int_{R^{n}}|\Phi(z, u+i v)|^{q} d u\right)^{1 / q} & \leq \text { const } \cdot\left(\int_{R^{n}}\left|\frac{e^{i<z+i v, t>}}{\gamma^{*}(2 t)}\right|^{p} d t\right)^{1 / p} \\
& =\text { const } \cdot\left(\int_{R^{n}} \frac{e^{-p<y+v, t>}}{\left[\gamma^{*}(2 t)\right]^{p}} d t\right)^{1 / p} \equiv R_{z}(v) .
\end{aligned}
$$

Hence (2.27) implies:

$$
\widetilde{I}(z) \leq \text { const } \cdot \int_{B} \widetilde{f}(v) R_{z}(v) \gamma(v) d v \equiv J .
$$

Note that the condition $f \in H_{s, \gamma}^{p}\left(T_{B}\right)$ implies

$$
\int_{B}[\tilde{f}(v)]^{p s} \gamma(v) d v<+\infty
$$

Moreover, in view of Proposition 2.2

$$
\sup _{v \in B}\left\{R_{z}(v)\right\}<+\infty .
$$

So, it remains to show $J<+\infty$ under conditions (2.31) and (2.32). To this end consider the measure $d \mu(v)=R_{z}(v) \gamma(v) d v(v \in B)$ and note that $\mu_{0}=\int_{B} d \mu(v)<+\infty$ due to $(2.32)$ and initial assumption $\gamma \in L^{1}(B ; d y)$. As a consequence, the integral can be written as

$$
J=\int_{B} \widetilde{f}(v) d \mu(v) .
$$


In view of the condition $1 / p \leq s<+\infty \Leftrightarrow p s \geq 1$ Hölder's integral inequality can be applied to (2.33) with $p s$ instead of $p$ and with $p s /(p s-1)$ instead of $q$ :

$$
\begin{aligned}
J=\int_{B} \tilde{f}(v) \cdot 1 d \mu(v) & \leq\left(\int_{B}[\tilde{f}(v)]^{p s} d \mu(v)\right)^{1 / p s} \cdot\left(\int_{B} 1 d \mu(v)\right)^{1 / q} \Rightarrow \\
J^{p s} & \leq \int_{B}[\tilde{f}(v)]^{p s} d \mu(v) \cdot \mu_{0}^{p s-1}<+\infty
\end{aligned}
$$

in view of (2.31)-(2.32). This completes the proof of the theorem.

3. Important special CASE

In this section we discuss the following particular case:

$$
\gamma(y)=\left(1-|y|^{2}\right)^{\alpha}, \quad y \in B(\alpha>-1)
$$

As it will be shown below, in this case $\gamma^{*}(t), t \in R^{n}$ can be evaluated more explicitly. Moreover, the estimates $(2.5),(2.6)$ and (2.19) can be strengthened.

We begin with several well-known facts.

The Bessel function $J_{\lambda}(z)(\lambda \in C)$ is defined as follows:

$$
\begin{aligned}
J_{\lambda}(z) & =\sum_{k=0}^{\infty} \frac{(-1)^{k} \cdot(z / 2)^{2 k+\lambda}}{\Gamma(k+1) \cdot \Gamma(k+1+\lambda)} \\
& \equiv(z / 2)^{\lambda} \cdot \sum_{k=0}^{\infty} \frac{(-1)^{k} \cdot(z / 2)^{2 k}}{\Gamma(k+1) \cdot \Gamma(k+1+\lambda)}, \quad z \in C .
\end{aligned}
$$

The important properties of $J_{\lambda}$ (when $\operatorname{Re} \lambda>-\frac{1}{2}$ ) are given by the following assertion $([20, \S 11],[21$, p.647]):

Theorem 3.1. (a) For any $z \in C$

$$
\begin{aligned}
J_{\lambda}(z) & =\frac{(z / 2)^{\lambda}}{\sqrt{\pi} \cdot \Gamma\left(\lambda+\frac{1}{2}\right)} \cdot \int_{-1}^{1} e^{i \tau z} \cdot\left(1-\tau^{2}\right)^{\lambda-\frac{1}{2}} d \tau \\
& \equiv \frac{2 \cdot(z / 2)^{\lambda}}{\sqrt{\pi} \cdot \Gamma\left(\lambda+\frac{1}{2}\right)} \cdot \int_{0}^{1} \cos (\tau z) \cdot\left(1-\tau^{2}\right)^{\lambda-\frac{1}{2}} d \tau .
\end{aligned}
$$

(b) For any $z \in C$

$$
J_{\lambda}(i z)=\frac{2 \cdot(i z / 2)^{\lambda}}{\sqrt{\pi} \cdot \Gamma\left(\lambda+\frac{1}{2}\right)} \cdot \int_{0}^{1} \operatorname{ch}(\tau z) \cdot\left(1-\tau^{2}\right)^{\lambda-\frac{1}{2}} d \tau .
$$

(c) When $\lambda>-\frac{1}{2}$, then

$$
J_{\lambda}(i x) \stackrel{\lambda}{\asymp} \frac{e^{x}}{\sqrt{x}}, x \rightarrow+\infty,
$$




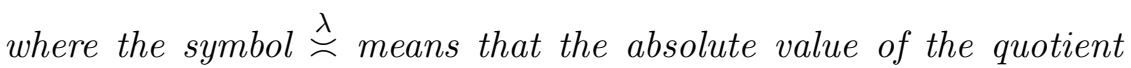
of corresponding expressions remains between two fixed positive constants (depending only on $\lambda$ ) when $x \rightarrow+\infty$.

Theorem 3.2. The following formulas are valid:

$$
\begin{aligned}
& \gamma^{*}(0)=\int_{B}\left(1-|y|^{2}\right)^{\alpha} d y=\frac{\pi^{\frac{n}{2}} \cdot \Gamma(\alpha+1)}{\Gamma\left(\alpha+\frac{n}{2}+1\right)}, \\
& \gamma^{*}(t)=\frac{\pi^{\frac{n}{2}} \cdot \Gamma(\alpha+1)}{(i|t| / 2)^{\alpha+\frac{n}{2}}} \cdot J_{\alpha+\frac{n}{2}}(i|t|), \quad t \in R^{n} \backslash\{0\}, \\
& \gamma^{*}(t) \stackrel{\alpha, n}{\longleftarrow} \frac{e^{|t|}}{|t|^{\alpha+\frac{n+1}{2}}},|t| \rightarrow+\infty .
\end{aligned}
$$

Proof. Introducing the polar coordinates in the integral of (3.6) we obtain:

$$
\begin{aligned}
\gamma^{*}(0) & =\sigma(S) \cdot \int_{0}^{1}\left(1-r^{2}\right)^{\alpha} r^{n-1} d r=\frac{\sigma(S)}{2} \cdot \int_{0}^{1}(1-t)^{\alpha} t^{\frac{n}{2}-1} d t \\
& =\frac{\sigma(S)}{2} \cdot B\left(\alpha+1 ; \frac{n}{2}\right)=\frac{\pi^{\frac{n}{2}}}{\Gamma\left(\frac{n}{2}\right)} \cdot \frac{\Gamma(\alpha+1) \Gamma\left(\frac{n}{2}\right)}{\Gamma\left(\alpha+1+\frac{n}{2}\right)}=\frac{\pi^{\frac{n}{2}} \cdot \Gamma(\alpha+1)}{\Gamma\left(\alpha+\frac{n}{2}+1\right)} .
\end{aligned}
$$

In order to establish (3.7) note that the function $\gamma^{*}(t)$ is radial, i.e. it depends, in fact, only on $|t|$ of the variable $t \in R^{n}$. Hence $\gamma^{*}\left(t_{1}, t_{2}, \ldots, t_{n}\right) \equiv$ $\gamma^{*}(|t|, 0, \ldots, 0)$ and, with the natural notation $y=\left(y_{1}, y_{2}, \ldots, y_{n}\right) \equiv\left(y_{1}, y^{\prime}\right)$ in the mind, we have:

$$
\begin{aligned}
\gamma^{*}(t) & =\int_{B} e^{-y_{1}|t|}\left(1-|y|^{2}\right)^{\alpha} d y=\int_{\left|y_{1}\right|<1} e^{-y_{1}|t|} \int_{\left|y^{\prime}\right|<\sqrt{1-y_{1}^{2}}}\left(1-y_{1}^{2}-\left|y^{\prime}\right|^{2}\right)^{\alpha} d y^{\prime} d y_{1} \\
& =\int_{\left|y_{1}\right|<1} e^{-y_{1}|t|}\left(1-y_{1}^{2}\right)^{\alpha+\frac{n-1}{2}} \int_{\omega \in C^{n-1},|\omega|<1}\left(1-|\omega|^{2}\right)^{\alpha} d \omega d y_{1}
\end{aligned}
$$

According to (3.6) the inner integral in (3.9) is equal to $\frac{\pi^{\frac{n-1}{2}} \cdot \Gamma(\alpha+1)}{\Gamma\left(\alpha+\frac{n+1}{2}\right)}$. Consequently,

$$
\begin{aligned}
\gamma^{*}(t) & =\frac{\pi^{\frac{n-1}{2}} \cdot \Gamma(\alpha+1)}{\Gamma\left(\alpha+\frac{n+1}{2}\right)} \cdot \int_{\left|y_{1}\right|<1} e^{-y_{1}|t|} \cdot\left(1-y_{1}^{2}\right)^{\alpha+\frac{n-1}{2}} d y_{1} \\
& =2 \frac{\pi^{\frac{n-1}{2}} \cdot \Gamma(\alpha+1)}{\Gamma\left(\alpha+\frac{n+1}{2}\right)} \cdot \int_{0}^{1} \operatorname{ch}(\tau|t|) \cdot\left(1-\tau^{2}\right)^{\alpha+\frac{n-1}{2}} d \tau \\
& =\pi^{\frac{n}{2}} \Gamma(\alpha+1) \cdot \frac{2}{\sqrt{\pi} \cdot \Gamma\left(\alpha+\frac{n}{2}+\frac{1}{2}\right)} \cdot \int_{0}^{1} \operatorname{ch}(\tau|t|) \cdot\left(1-\tau^{2}\right)^{\left(\alpha+\frac{n}{2}\right)-\frac{1}{2}} d \tau .
\end{aligned}
$$


On the other hand, according to (3.4) we have:

$$
J_{\alpha+\frac{n}{2}}(i|t|)=\frac{2 \cdot(i|t| / 2)^{\alpha+\frac{n}{2}}}{\sqrt{\pi} \cdot \Gamma\left(\alpha+\frac{n}{2}+\frac{1}{2}\right)} \cdot \int_{0}^{1} \operatorname{ch}(\tau|t|) \cdot\left(1-\tau^{2}\right)^{\left(\alpha+\frac{n}{2}\right)-\frac{1}{2}} d \tau
$$

The comparison of (3.10) and (3.11) yields (3.7).

Further, (3.5) implies:

$$
J_{\alpha+\frac{n}{2}}(i|t|) \asymp \frac{e^{|t|}}{|t|^{\frac{1}{2}}},|t| \rightarrow+\infty .
$$

Combining (3.12) with (3.7), we obtain (3.8).

Remark 3.1. Note that in the special case under consideration the asymptotic formula (3.8) describes the behaviour of $\gamma^{*}(t)$ (when $t \rightarrow+\infty$ ) much more explicitly than the estimates (2.5) and (2.6) of Proposition 2.1.

The following assertion gives more explicit estimate for the kernel $\Phi(z, w)$ $(\operatorname{see}(2.17))$ :

Theorem 3.3. If $0<\delta<2$, then

$$
|\Phi(z, w)| \leq \operatorname{const}(\alpha ; n)+\frac{\operatorname{const}(\alpha ; n)}{\delta^{\frac{3 n+1}{2}+\alpha}}
$$

uniformly in $z=x+i y, w=u+i v \in T_{B}$ with $|v|+|y| \leq 2-\delta$.

Proof. First of all, note (see the proof of Proposition 2.3) that under the assumption $|v|+|y| \leq 2-\delta$ with any $\delta \in(0 ; 2)$ we have:

$$
\left|\frac{e^{i<z-\bar{w}, t>}}{\gamma^{*}(2 t)}\right| \leq \frac{e^{(2-|\delta|)|t|}}{\gamma^{*}(2 t)}
$$

Hence

$$
|\Phi(z, w)| \leq \int_{R^{n}} \frac{e^{(2-|\delta|)|t|}}{\gamma^{*}(2 t)} d t=\int_{|t| \leq 1} \ldots d t+\int_{|t| \geq 1} \ldots d t \equiv I_{1}+I_{2} .
$$

In view of (3.7)

$$
I_{1} \leq \operatorname{const}(\alpha ; n) .
$$


As to the integral $I_{2}$, due to (3.8) and (3.7) we have:

$$
\begin{aligned}
I_{2} & \leq \operatorname{const}(\alpha ; n) \cdot \int_{|t| \geq 1} \frac{e^{(2-|\delta|)|t|} \cdot|t|^{\frac{n+1}{2}+\alpha}}{e^{2|t|}} d t \\
& =\operatorname{const}(\alpha ; n) \cdot \int_{|t| \geq 1} e^{-|\delta||t|} \cdot|t|^{\frac{n+1}{2}+\alpha} d t \\
& =\operatorname{const}(\alpha ; n) \cdot \int_{1}^{+\infty} e^{-|\delta| r} \cdot r^{\frac{n+1}{2}+\alpha+n-1} d r \stackrel{\delta r \rightarrow r}{=}= \\
& =\frac{\operatorname{const}(\alpha ; n)}{\delta^{\frac{n+1}{2}+\alpha+n}} \cdot \int_{\delta}^{+\infty} e^{-r} \cdot r^{\frac{n+1}{2}+\alpha+n-1} d r \\
& \leq \frac{\operatorname{const}(\alpha ; n)}{\delta^{\frac{n+1}{2}+\alpha+n}} \cdot \Gamma\left(\frac{n+1}{2}+\alpha+n\right) \equiv \frac{\operatorname{const}(\alpha ; n)}{\delta^{\frac{n+1}{2}+\alpha+n}}
\end{aligned}
$$

Combination of (3.16) and (3.17) gives (3.13). The proof is completed.

Remark 3.2. Note that under the additional restriction $0<\delta \leq 1$ the estimate (3.13) can be written as follows:

$$
|\Phi(z, w)| \leq \frac{\operatorname{const}(\alpha ; n)}{\delta^{\frac{3 n+1}{2}+\alpha}} .
$$

\section{REFERENCES}

[1] R. Paley and N. Wiener, Fourier transforms in the complex plane, Amer. Math. Soc. Colloq. Publ., 19, Amer. Math. Soc., Providence, R.I. 1934.

[2] M. M. Djrbashian and A. E. Avetisian, Integral representations of some classes of functions analytic in an angular domain, Dokl. Akad. Nauk SSSR, 120 (3) (1958), 457-460 (in Russian).

[3] M. M. Djrbashian, Integral transforms and representations of functions in a complex domain, Nauka, Moscow, 1966 (in Russian).

[4] S. Bochner, Group invariance of Cauchy formula in several variables, Ann. Math., 45 (1944), 686-707.

[5] S. Bochner and W. T. Martin, Several Complex Variables, Princeton Univ. Press, Princeton, New York, 1948.

[6] S. G. Gindikin, Analysis in homogeneous domains, Uspekhi Mat. Nauk 19 (4) (1964), 3-92 (in Russian).

[7] T. G. Genchev, Paley-Wiener type theorems for functions in a half-plane, Dokl. Bulg. Akad. Nauk, 37 (1983), 141-144.

[8] T. G. Genchev, Integral representations for functions holomorphic in tube domains, Dokl. Bulg. Akad. Nauk, 37 (1984), 717-720.

[9] T. G. Genchev, Paley-Wiener type theorems for functions in Bergman spaces over tube domains, J. Math. Anal. Appl., 118 (1986), 496-501.

[10] M. M. Djrbashian and V. E. Martirosian, Integral representations for some classes of functions holomorphic in a strip or in a half-plane, Dokl. Akad. Nauk SSSR, 283 (5) (1985), 1054-1057 (in Russian). 
[11] A. H. Karapetyan, Some questions of integral representations in multidimensional complex analysis, Candidate Dissertation, Yerevan, 1987 (in Russian).

[12] A. H. Karepetyan, Integral representations in tube domains, J. Contemp. Math. Anal., 23 (1) (1988), 90-95.

[13] A. H. Karepetyan, Integral representations for weighted spaces of functions holomorphic in tube domains, J. Contemp. Math. Anal., 254 (4) (1990),1-19.

[14] A. H. Karepetyan, Integral representations of holomorphic functions in radial tube domains, J. Contemp. Math. Anal., 26 (1) (1991), 1-25.

[15] A. H. Karepetyan, Integral representations in tube domains over affine-homogeneous cones, J. Contemp. Math. Anal., 27 (1) (1992), 1-21.

[16] A. H. Karapetyan, Integral representations in tube domains over polyhedra and questions of function approximations, Anal. Math., 20 (3) (1994), 185-203 (in Russian).

[17] S. Saitoh, Generalizations of Paley-Wiener theorem for entire functions of exponential type, Proc. Amer. Math. Soc., 99 (1987), 465-471.

[18] S. Saitoh, Fourier-Laplace transforms and the Bergman spaces, Proc. Amer. Math. Soc., 102 (1988), 985-992.

[19] S. Saitoh, Integral transforms, reproducing kernels and their applications, Pitman Research Notes in Math., 369, Longman, UK, 1997.

[20] J. Kampe de Feriet, Fonctions de la Physique Mathematique, Centre National de la Recherche Scientifique, 1957.

[21] M. A. Lavrentev and B. V. Shabat, Methods of the theory of functions of complex variable, Nauka, Moscow, 1965 (in Russian).

(Received: April 9, 2015)

(Revised: April 24, 2015)
Institute of Mathematics

National Academy of Sciences of Armenia

Marshal Bagramian Ave.24/5

Yerevan 0019

Armenia

armankar2005@rambler.ru 